\title{
The influence of dietary energy restriction and compensatory growth on metabolic and contractile muscle characteristics in bovine
}

\author{
A Brandstetter 2, B Picard 1, Y Geay 1 \\ 1/NRA, Croissance et Métabolismes des Herbivores, Theix, 63122 St-Genès-Champanelle, France ; ZUniversity \\ of Agriculture Vienna, Department of Livestock Production, Gregor-Mende/str 33, 180 Vienna, Austria
}

Metabolic and contractile activity of muscle fibers play a fundamental role in modelling subsequently meat quality and tenderness, affecting meat traits during postmortem evolution (Valin, 1988, Reprod Nutr Dev, 28, 845-856). Thus, a serial slaughter experiment was conducted to study the development of myofibrillar metabolic and contractile characteristics during growth and to determine their modification by a nutritionally imposed period of growth restriction followed by an accelerated growth rate due to realimentation.

Thirty Montbéliard bulls, bought at 2 weeks of age, received after weaning (at 4 months) the same diet at the same live weight. The animals were allowed to grow at approximately 1100 $\mathrm{g} / \mathrm{d}$ up to 9 months. At 4 and 8 months of age, 5 representative animals were slaughtered. At 9 months the remaining 20 bulls were assigned to two groups of 10 , according to their weight and previous growth rate. The bulls of the first group continued to receive the same diet ad libitum (NR) and 5 animals were respectively slaughtered at 12 and 16 months. The second group was restricted (R) from 9 to 12 months to realize about $60 \%$ of the control daily gain ( $737 \mathrm{~g} / \mathrm{d}$ vs $1295 \mathrm{~g} / \mathrm{d}$ ) and then fed ad libitum to express compensatory growth $(1410 \mathrm{~g} / \mathrm{d}$ vs $1080 \mathrm{~g} / \mathrm{d}$ ). Five animals respectively were slaughtered at 12 and 16 months of age. Muscle samples of different anatomical regions and varying myofiber composition, i.e. Semitendinosus (ST), Triceps Brachii (TB), Biceps Femoris (BF) and Longissimus Dorsi pars thoracis (LD) have been removed $24 \mathrm{~h}$ post mortem. Enzymatic kinetics for isocitrate dehydrogenase (ICDH ; aerobic metabolism) and for lactate dehydrogenase (LDH ; anaerobic metabolism) were measured spectrophotometrically. Contractile muscle type was classified by quantification of myosin heavy chain I isoform ( $\mathrm{MHCl}$ ) proportion using the sensitive enzyme-linked immunosorbent assay (ELISA) (Jurie et al, 1995, Meat Sc, $39,3,415-427)$.

The level and chronological evolution of metabolic activities is muscle-dependent with generally a rapid increase in glycolytic function during initial growth phase and little changes in oxidative capacity. At 12 months nutritionally restricted $(R)$ bulls showed higher ICDH-levels than NR bulls (ST : 0.295 vs 0.226 nkat (= nmoles/s)/g muscle), this in coherence with elevated $\mathrm{MHCl}$-proportions (ST : $32.7 \%$ vs $27.4 \%$ ) and lower LDH activities (ST : 108.2 vs $115.5 \mathrm{nkat} / \mathrm{g}$ muscle), though not significant. Realimentation succeeding the period of undernutrition led to the inversion of the previously observed adaptation. At the age of 16 months, R bulls, compared to NR bulls at the same age, showed significantly lower ICDH-activities in the LT muscle $(0.128$ vs $0.207 \mathrm{nkat} / \mathrm{g}$ muscle) and a trend towards reduced oxidative metabolism in the ST and $\mathrm{BF}$ muscles, tendentiously paralleled by lower $\mathrm{MHCl}$-proportions as well as superior $\mathrm{LDH}$-activity.

The reduction of feeding level induces more oxidative muscles due not only to a shifting towards IIA (oxido-glycolytic) fibers at the expense of IIB (glycolytic) fibers, but also to an increased occurrence of type I fibers. Conversely, compensatory growth leads to a metabolic orientation in favour of glycolytic activity with a decrease in slow oxidative fibers (type I). This could be favourable to an improvement of meat tenderness, since fast glycolytic fibers have a higher rate of ageing than the slow ones (Ouali, 1990, J Muscle Foods, 1, 129-165). 